\title{
Consensus statements from the EAU-ESMO collaboration for advanced and variant bladder cancer: can we move the needle to improve survival?
}

\author{
Vignesh T. Packiam \\ Department of Urology, University of Iowa, Iowa City, IA, USA \\ Correspondence to: Vignesh T. Packiam. Department of Urology, University of Iowa, 3 RCP, 200 Hawkins Drive, Iowa City, IA 52242, USA. \\ Email: vignesh-packiam@uiowa.edu. \\ Comment on: Witjes JA, Babjuk M, Bellmunt J, et al. EAU-ESMO consensus statements on the management of advanced and variant bladder cancer- \\ an international collaborative multistakeholder effort $†$ : under the auspices of the EAU-ESMO Guidelines Committees. Eur Urol 2020;77:223-50.
}

Submitted May 20, 2020. Accepted for publication Jun 19, 2020.

doi: $10.21037 /$ tau-20-934

View this article at: http://dx.doi.org/10.21037/tau-20-934

Bladder cancer caused an estimated 17,980 deaths in the United States in 2020 (1). Unfortunately, death from bladder cancer has not decreased over the last few decades, with an estimated 12,200 deaths in 2000, a trend corroborated by independent analysis $(2,3)$. Over the past years we have witnessed an explosion of advances in bladder cancer treatment, spanning from novel bladdersparing treatments for non-muscle invasive disease to the development and rapid adoption of immune checkpoint inhibition for advanced stages $(4,5)$. How do we harness these advances to move the needle and improve oncologic outcomes for bladder cancer? One facet will be to contextualize all advances across specialties rather than viewing them in isolation from a single standpoint. Advances in the management of non-muscle invasive disease, trimodal therapy, and immune checkpoint inhibition should not be relegated exclusively to the domains of urologists, radiation oncologists, and medical oncologists, respectively. To improve outcomes for patients with bladder cancer, inclusive multi-specialty collaboration is required to synthesize a succinct and centralized best practice resource.

The recent EAU-ESMO Consensus statements by Witjes et al. on the management of advanced and variant bladder cancer is a colossal and timely international multi-specialty effort (6). The authors utilized Delphi methodology to draw from 134 experts from 23 countries, comprised of urologists, oncologists (including medical and radiation oncologists), and "other" stakeholders (including radiologists, pathologists, specialist nurses, clinical oncologists, and nuclear medicine specialists). While the entirety of the 143 consensus statements warrant careful review, several statements are of particular interest and importance across the landscape of bladder cancer.

\section{Variant histology}

First, considering non-muscle invasive bladder cancer, there are several salient points made regarding urothelial histologic variants. There was $86 \%$ agreement for the consensus statement advocating immediate radical cystectomy and lymphadenectomy for patients with T1 urothelial carcinoma (UC) with variant micropapillary histology. The strength of this recommendation contrasts the equipoise demonstrated in a recent systematic review and meta-analysis, which highlights more controversy in considering bladder-sparing management strategies for some cases of bladder UC with limited proportion, extent, and depth of micropapillary component (7). Recommendations regarding the rarer plasmacytoid, sarcomatoid, and nested variants showed more variability, with $64 \%$ of urologists, $52 \%$ of oncologists, and $18 \%$ of other stakeholders advocating for immediate radical cystectomy. Particularly for the plasmacytoid variant, due to its predilection for aggressive and diffuse local invasion with characteristically indiscernible radiologic characteristics, immediate extirpative surgery for clinical $\mathrm{T} 1$ disease is warranted (8). 
Regarding pure divergent muscle-invasive histologies, there was near universal agreement to administer neoadjuvant chemotherapy potentially followed by local consolidative surgery for small cell neuroendocrine variants, and to proceed with primary radical cystectomy and lymphadenectomy for squamous cell carcinoma and adenocarcinoma. For UC with variant histologies, there was less agreement. Only $63 \%$ recommended neoadjuvant chemotherapy for micropapillary, plasmacytoid, squamous or glandular differentiation histologies. The lack of consensus in the paper results from numerous competing issues of variant histologies. First, the clear association with variant histology and clinical understaging with respect to local extension and micrometastatic disease supports consideration for neoadjuvant systemic therapy in addition to timely cystectomy. Second, aberrant tumor biology and the presence of tumor heterogeneity may also reduce the efficacy of systemic treatments (9). Additionally, each of these variant histologies possesses variable response rates to systemic treatments. Molecular sequencing, subtyping, and profiling are currently under investigation and may supplement the predominant use of clinical factors and immunohistochemistry for management decisions. As a final note, it is important to stress that optimal decision making for histologic variants hinges on a meticulous and highquality transurethral resection of bladder tumor with expert pathologic review $(10,11)$.

\section{Neoadjuvant therapy}

While the overall survival benefit of neoadjuvant cisplatinbased chemotherapy for patients with muscle-invasive bladder cancer is well established, there does exist a distinct subset of patients who do not respond $(12,13)$. Thus, effective patient selection is critical to determine who benefits from immediate radical cystectomy versus surgery preceded by neoadjuvant therapy. For example, the consensus paper highlights a landmark study by Seiler et al. that recommends molecular subtyping with prioritization of neoadjuvant chemotherapy for patients with basal tumors (14). While some data supports using biomarkers to guide neoadjuvant systemic therapy, prospective validation is needed (15). Prognostic molecular markers certainly hold promise, but the authors conclude that biomarkers are not yet ready for prime time. Regarding neoadjuvant immunotherapy, there is promising data from the PURE01 and ABACUS trials for neoadjuvant pembrolizumab and Atezolizumab, respectively, but these agents have not been validated in phase III studies $(16,17)$. Therefore, there was strong consensus to not use neoadjuvant immune checkpoint inhibitors outside of a clinical trial.

\section{Bladder-sparing approaches and pelvic lymphadenectomy}

Trimodal therapy for muscle invasive disease appears to be gaining traction in some areas but interest is noted to be geographically specific, particularly with respect to country. Specialist bias may play a large role in patient selection, and for this reason, the bladder-sparing consensus statement recommending multidisciplinary consultation with a urologist, oncologist and a neutral party such as a specialist nurse, had $83 \%$ agreement. Advocacy groups such as the Bladder Cancer Advocacy Network (BCAN) can help serve a key role by connecting patients to others who have previously undergone these treatments. While most of the technical aspects of radiosensitizing chemotherapy and radiotherapy techniques had strong consensus, the role of pelvic lymphadenectomy within bladder-sparing algorithms provides some controversy. Only 64\% agreed to not perform pelvic lymphadenectomy for clinically node negative muscle invasive disease. Interestingly, "other" stakeholders (83\%) supported the use of pelvic lymphadenectomy compared to urologists $(47 \%)$ and oncologists $(25 \%)$. This is likely secondary to different perceptions of patient selection between types of specialists, as urologists note that well selected trimodal therapy patients often have less aggressive disease, with lower risk of nodal metastases. Further highlighting the potential for selection bias, it was noted that less than $10 \%$ of patients had lymph node relapse in the BC2001trial, which is lower than the $25 \%$ node positive rate typically seen in large cystectomy series (18).

\section{Oligometastatic disease}

The EAU-ESMO Consensus statement was the first to discuss the nuances of surgical selection for oligometastatic disease, distinguishing it from prior guidelines in this space. There was strong $91 \%$ consensus that cure is possible in some patients with a single metastatic lesion. There was less consensus for patients with two metastatic lesions as supported by retrospective data and a recent systematic review (19). When stratified by specific metastatic site, lymph node only disease was deemed most favorable, followed by lung, liver, and bone. 
There were also comments regarding the role of PETCT for patients in consideration of radical treatment for oligometastatic disease. While $88 \%$ agreed that PET-CT should be performed for these patients, the data for this is relatively limited and requires further study (20). Most agreed with the intuitive concept that radical treatment of oligometastatic disease should be accompanied by systemic therapy. However, there was less clarity regarding optimal type and duration of systemic regimens. A particular conundrum is for isolated clinically node positive disease, where some retrospective data suggests benefit from longer induction regimens over shorter neoadjuvant courses, although this lacks prospective validation (21).

\section{Immune checkpoint inhibition}

Regarding immune checkpoint inhibition, the most definitive consensus was achieved regarding the lack of pseudoprogression observed for UC, therefore necessitating that radiographic progression on checkpoint inhibition should be promptly followed by a switch to chemotherapy. The role of sequencing and combining chemotherapy and immunotherapy is currently being assessed by a myriad of trials. While this paper demonstrates relatively strong consensus against combination chemotherapy and immunotherapy for metastatic disease, this has the potential to rapidly change, as a study was just recently published demonstrating the efficacy of combination Atezolizumab with platinum-based chemotherapy for first line metastatic disease (22). Ultimately, the authors wisely recommend supporting clinical trials to allow for the safe adoption of rapidly changing available treatment regimens.

\section{Consensus process}

The importance of an inclusive multi-disciplinary process was shown by several examples of consensus changes between the initial Delphi survey and post-conference consensus statements. This reflects the presence of uncertainty and lack of definitive authority from a single specialty. For example, $80 \%$ of urologists initially disagreed with stopping regular surveillance after 5 years following cystectomy, while $88 \%$ of participants agreed to stop regular surveillance after this time on the final consensus statement. The viewpoint that was lacking in the paper was the patient perspective, which is of particular importance when considering the psychosocial implications for followup and survivorship. This can be better voiced with the inclusion of patient advocacy groups.

\section{Future directions}

In summary, the consensus statements provided under the direction of the EAU-ESMO Committees is an invaluable resource for physicians managing bladder cancer. There will inevitably be a need to amend some of these recommendations as advances rapidly continue to arrive. For example, the role of checkpoint inhibition for non-muscle invasive bladder cancer, while not supported in these consensus statements, has rapidly shifted with the recent FDA approval for Pembrolizumab following the KEYNOTE-057 trial (23). There are many other questions that remain to be answered and can further improve oncologic outcomes. The role of MRI imaging and the VIRADS system for bladder cancer is intriguing, with implications for both non-muscle invasive and muscleinvasive disease (24). Patient selection for neoadjuvant chemotherapy needs further optimization based on staging, variant histology, and molecular profiling. Optimizing management of clinically node positive disease is currently understudied. The role of both imaging, such as PETCT, and biomarkers, such as circulating tumor cells, for restaging during neoadjuvant treatment and for detection of metastatic and recurrent disease, needs to be further investigated. Finally, the role of bladder sparing approaches, both for initial management of muscle invasive disease and in the setting of clinical response following systemic therapy, requires careful evaluation. Ultimately, further studies and the effective dissemination of their results will allow the needle to move, improving oncologic outcomes and quality of life for patients with bladder cancer.

\section{Acknowledgments}

Funding: None.

\section{Footnote}

Provenance and Peer Review: This article was commissioned by the editorial office, Translational Andrology and Urology. The article did not undergo external peer review.

Conflicts of Interest: The author has completed the ICMJE uniform disclosure form (available at http://dx.doi. org/10.21037/tau-20-934). The author has no conflicts of interest to declare. 
Ethical Statement: The author is accountable for all aspects of the work in ensuring that questions related to the accuracy or integrity of any part of the work are appropriately investigated and resolved.

Open Access Statement: This is an Open Access article distributed in accordance with the Creative Commons Attribution-NonCommercial-NoDerivs 4.0 International License (CC BY-NC-ND 4.0), which permits the noncommercial replication and distribution of the article with the strict proviso that no changes or edits are made and the original work is properly cited (including links to both the formal publication through the relevant DOI and the license). See: https://creativecommons.org/licenses/by-nc-nd/4.0/.

\section{References}

1. Siegel RL, Miller KD, Jemal A. Cancer statistics, 2020. CA Cancer J Clin 2020;70:7-30.

2. Greenlee RT, Murray T, Bolden S, et al. Cancer statistics, 2000. CA Cancer J Clin 2000;50:7-33.

3. Abdollah F, Gandaglia G, Thuret R, et al. Incidence, survival and mortality rates of stage-specific bladder cancer in United States: a trend analysis. Cancer Epidemiol 2013;37:219-25.

4. Packiam VT, Werntz RP, Steinberg GD. Current clinical trials in non-muscle-invasive bladder cancer: heightened need in an era of chronic BCG shortage. Curr Urol Rep 2019;20:84.

5. Boormans JL, Zwarthoff EC, Black PC, et al. New horizons in bladder cancer research. Urol Oncol 2020;38:867-85

6. Witjes JA, Babjuk M, Bellmunt J, et al. EAU-ESMO Consensus statements on the management of advanced and variant bladder cancer-an international collaborative multistakeholder effort + : under the auspices of the EAUESMO Guidelines Committees. Eur Urol 2020;77:223-50.

7. Abufaraj M, Foerster B, Schernhammer E, et al. Micropapillary urothelial carcinoma of the bladder: a systematic review and meta-analysis of disease characteristics and treatment outcomes. Eur Urol 2019;75:649-58.

8. Fox MD, Xiao L, Zhang M, et al. Plasmacytoid urothelial carcinoma of the urinary bladder: a clinicopathologic and immunohistochemical analysis of 49 cases. Am J Clin Pathol 2017;147:500-6.

9. Meeks JJ, Al-Ahmadie H, Faltas BM, et al. Genomic heterogeneity in bladder cancer: challenges and possible solutions to improve outcomes. Nat Rev Urol 2020;17:259-70.

10. Anderson C, Weber R, Patel D, et al. A 10-item checklist improves reporting of critical procedural elements during transurethral resection of bladder tumor. J Urol 2016;196:1014-20.

11. Mostafid H, Babjuk M, Bochner B, et al. Transurethral resection of bladder tumour: the neglected procedure in the technology race in bladder cancer. Eur Urol 2020;77:669-70.

12. Grossman HB, Natale RB, Tangen CM, et al. Neoadjuvant chemotherapy plus cystectomy compared with cystectomy alone for locally advanced bladder cancer. $\mathrm{N}$ Engl J Med 2003;349:859-66.

13. Bhindi B, Frank I, Mason RJ, et al. Oncologic outcomes for patients with residual cancer at cystectomy following neoadjuvant chemotherapy: a pathologic stage-matched analysis. Eur Urol 2017;72:660-4.

14. Seiler R, Ashab HAD, Erho N, et al. Impact of molecular subtypes in muscle-invasive bladder cancer on predicting response and survival after neoadjuvant chemotherapy. Eur Urol 2017;72:544-54.

15. Grossman HB, Bellmunt J, Black PC. Can biomarkers guide the use of neoadjuvant chemotherapy in T2 bladder cancer? Eur Urol Oncol 2019;2:597-602.

16. Necchi A, Anichini A, Raggi D, et al. Pembrolizumab as neoadjuvant therapy before radical cystectomy in patients with muscle-invasive urothelial bladder carcinoma (PURE01): an open-label, single-arm, phase II study. J Clin Oncol 2018;36:3353-60.

17. Powles T, Rodriguez-Vida A, Duran I, et al. A phase II study investigating the safety and efficacy of neoadjuvant atezolizumab in muscle invasive bladder cancer (ABACUS). J Clin Oncol 2018;36:4506.

18. Stein JP, Lieskovsky G, Cote R, et al. Radical cystectomy in the treatment of invasive bladder cancer: long-term results in 1,054 patients. J Clin Oncol 2001;19:666-75.

19. Abufaraj M, Dalbagni G, Daneshmand S, et al. The role of surgery in metastatic bladder cancer: a systematic review. Eur Urol 2018;73:543-57.

20. Kibel AS, Dehdashti F, Katz MD, et al. Prospective study of [18F] fluorodeoxyglucose positron emission tomography/computed tomography for staging of muscle-invasive bladder carcinoma. J Clin Oncol 2009;27:4314-20.

21. Zargar-Shoshtari K, Zargar H, Lotan Y, et al. A multiinstitutional analysis of outcomes of patients with clinically node positive urothelial bladder cancer treated with 
induction chemotherapy and radical cystectomy. J Urol 2016;195:53-9.

22. Galsky MD, Arija JÁA, Bamias A, et al. Atezolizumab with or without chemotherapy in metastatic urothelial cancer (IMvigor 130): a multicentre, randomised, placebocontrolled phase 3 trial. Lancet 2020;395:1547-57.

23. Balar AV, Kulkarni GS, Uchio EM, et al. Keynote 057: Phase II trial of Pembrolizumab (pembro) for patients (pts) with high-risk (HR) nonmuscle invasive bladder cancer (NMIBC) unresponsive to bacillus calmette-guérin (BCG). J Clin Oncol 2019;37:350.

24. Panebianco V, Narumi Y, Altun E, et al. Multiparametric magnetic resonance imaging for bladder cancer: development of VI-RADS (vesical imaging-reporting and data system). Eur Urol 2018;74:294-306.
Cite this article as: Packiam VT. Consensus statements from the EAU-ESMO collaboration for advanced and variant bladder cancer: can we move the needle to improve survival? Transl Androl Urol 2020;9(6):2488-2492. doi: 10.21037/tau-20-934 